\title{
EQUIVALENT CHANGES/DIFFERENCES FOR PERCENTAGES AND PROBABILITIES
}

\author{
BY \\ YEW-KWANG NG \\ Monash University, Australia
}

\begin{abstract}
For percentages and probabilities or other fractions that have to lie between zero and one, it is not legitimate to take equal proportionate or absolute differences for two variables to register equivalent changes, since this may require one variable to exceed one. Reasonable properties for equivalent changes including Interchangeability, Parity, Identity, Differentiability, Monotonicity, Complementarity, Anonymity, and Transitivity are proposed. A specific function satisfying all these properties is provided and the question of the uniqueness of this function is discussed. The acceptance of the proposed function may lead to widespread practical application.
\end{abstract}

1. Introduction. Suppose variable $x$ changes from $x_{1}$ to $x_{2}$. Given $y_{1}$, what is the change in $y$ (i.e., for what value of $y_{2}$ ) can we say that $y$ changes by an equivalent extent as $x$ ? A simple answer is to take $y_{2} / y_{1}=x_{2} / x_{1}$, i.e., equivalent proportionate change. This works well for most cases where $x$ and $y$ are both positive absolute values, e.g., GNP of two countries. If $x$ and $y$ are probabilites or percentages or other fractions that have to lie between zero and one, the method of taking proportionate changes to indicate equivalent changes is no longer valid. To see this, suppose $x_{1}=1 \%, y_{1}=20 \%$. If $x_{2}=10 \%, y_{2}$ has to equal $200 \%$ for it to satisfy $y_{2} / y_{1}=x_{2} / x_{1}$. This is obviously impossible since $x$ and $y$ cannot exceed $100 \%$.

Equivalent change is an interesting concept since it allows us to say whether the actual change is smaller or larger (in one variable more than another). As far as I know, equivalent changes in percentages and probabilities have not been analyzed, despite their frequent occurrences in many fields. It may be noted that the concept of equivalent changes differ from that on measures of association for cross classifications as analysed, e.g., by Edwards [1] and Goodman and Kruskal [2].

Of course, there could be different concepts and measures of equivalent changes. For example, it may be very difficult, due to certain structural reasons, for youth unemployment to be reduced beyond, say, $15 \%$, while it is relatively easy for adult unemployment to be reduced to as low as $3-5 \%$. Then, in the sense of equivalent Received April 27, 1990.

Key words and phrases. Equivalent changes, equivalent differences, percentages, probabilities.

(C) 1991 Brown University 
difficulty, it may be reasonable to say that reducing youth unemployment from $15 \%$ to $14 \%$ is equivlent to the reduction of adult unemployment from $15 \%$ to $7 \%$. It is also clear that, since both youth and adult unemployment start from 15\% $\left(x_{1}=y_{1}=\right.$ $15 \%$ ), they must decrease or increase to the same figure to register equivalent changes in some formal or general sense. It is this general sense of equivalent changes that we are interested in, since we cannot cater to specific difficulties or other peculiarities associated with specific variables under specific circumstances. For such specific factors, one has to be contented in saying, e.g., that it is a greater feat to reduce youth unemployment from $15 \%$ to $12 \%$ though this registers a far less than an equivalent reduction in adult unemployment from $15 \%$ to $7 \%$.

The next section lists a dozen reasonable properties for equivalent changes for (positive) proper fractions (percentages and probabilties). Section III provides a specific function for $y_{2}$ given and $x_{1}, y_{1}, x_{2}$ (between zero and one), satisfying all these properties. Section IV discusses whether the function is unique and outlines areas for further research.

II. Reasonable properties required. Our problem is to find a value of $y_{2}$ for each and every combination of $x_{1}, y_{1}, x_{2}$, where all these variables lie between (and inclusive of) zero and one, and the change in (or difference of) $y$ from $y_{1}$ to $y_{2}$ is equivalent to that of $x$ from $x_{1}$ to $x_{2}$ in some general sense. In other words, we want to find a function $y_{2}=F\left(x_{1}, y_{1}, x_{2}\right)$ with domain the unit cube and range the unit interval $(0,1)$. There are some reasonable properties that we want the function $y_{2}=F\left(x_{1}, y_{1}, x_{2}\right)$ to satisfy:

Property 1 (Completeness). $F(\cdot)$ exists for all values of $x_{1}, y_{1}, x_{2}$ between, and inclusive of zero and one.

Property 2 (Uniqueness). For each and every value of $\left(x_{1}, y_{1}, x_{2}\right), F(\cdot)$ is unique.

If $\bar{y}_{2}$ is the value giving an equivalent change in $y$, given $y_{1}, x_{1}$, and $x_{2}$, we cannot have another value of $y_{2} \neq \bar{y}_{2}$ that also gives an equivalent change. It is this property that makes $F(\cdot)$ a function instead of just a correspondence.

Property 3 (InTerchangeability). $F\left\{x_{2}, F\left(x_{1}, y_{1}, x_{2}\right), x_{1}\right\}=y_{1}$. In other words, if we interchange the value of $x_{1}$ and $x_{2}$, and interchange the value of $y_{1}$ and $y_{2}$, this should not alter the original equivalence.

PROPERTY 4 (PARITY). $F\left(x_{1}, y_{1}, x_{2}\right)=x_{2}$ for all $x_{1}=y_{1}$ and all $x_{2}$ (between zero and one).

The compelling nature of this property is obvious. If $x$ and $y$ are equal to begin with, $y$ must change by the same amount as $x$ for it to be an equivalent change. This gives us the identity function $y=x$ as a special case that can be used to obtain a $y_{2}$ for any $x_{2}$, given $x_{1}=y_{1}$. This is represented by Curve $1\left(45^{\circ}\right.$ straight line $)$ in Fig. 1.

Property 5 (IDENTity). $F\left(x_{1}, y_{2}, x_{2}\right)=y_{1}$ if $x_{2}=x_{1}$.

If $x$ remains unchanged, $y$ has obviously to remain unchanged for equivalence. This means that the function $y_{2}=F\left(\bar{x}_{1}, \bar{y}_{1}, x_{2}\right) \equiv f^{i}\left(x_{2}\right)$ has to pass through the 


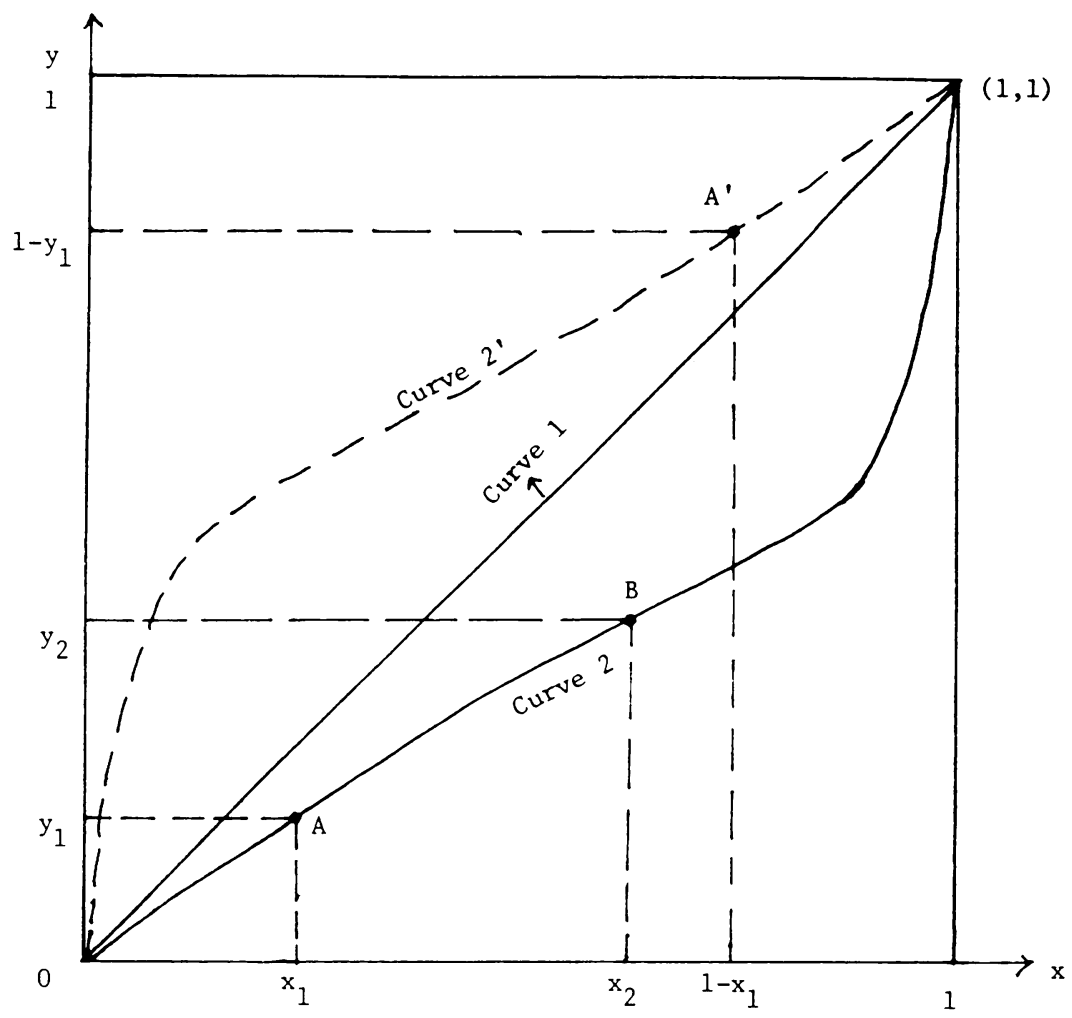

FIG. 1

point $\left(\bar{x}_{1}, \bar{y}_{1}\right)$ in the two-dimensional space with the $x$-axis measuring both $x_{1}$ and $x_{2}$ and the $y$-axis measuring both $y_{1}$ and $y_{2}$. This allows us to transform the problem of finding $y_{2}=F\left(x_{1}, y_{1}, x_{2}\right)$ for all $0 \leq x_{1}, y_{1}, x_{2} \leq 0$ into the following simpler form. Find a family of functions $y=f^{i}(x)$ where the specific $f^{i}$ is determined by the point $\left(x_{1}, y_{1}\right)$ in the two-dimensional unit space such that $y=f^{i}(x)$ passes through $x_{1}, y_{1}$ and the whole family of these functions covers the whole unit space.

Property 6 (Limitation). $F\left(x_{1}, y_{1}, 0\right)=0 ; F\left(x_{1}, y_{1}, 1\right)=1$.

This follows from the fact that neither $x$ nor $y$ can exceed one or fall below 0 . Thus, if $x$ increases to the upper limit (one), $y$ should and can only increase to one (to register an equivalent change). Similarly, if $x$ falls to the lower limit, $y$ should and can only fall to zero. This requirement is similar to the case where $x$ and $y$ are absolute numbers not confined to zero and one. If $x$ increases (falls) to infinity (zero or minus infinity as the case may be), $y$ should increase (fall) to infinity (zero, minus infinity) to register an equivalent change, irrespective of where it starts from.

A curve (e.g., Curve 2 in Fig. 1) passing through the points $(0,0)$ and $(1,1)$ satisfies Property 6 . Then, for any $\left(x_{1}, y_{1}\right)$ on the curve, it gives the corresponding $y_{2}$ for any $x_{2}\left(0 \leq x_{2} \leq 1\right)$, e.g., point $B$. However, since only a vanishing proportion (of measure zero) of all possible $\left(x_{1}, y_{1}\right)$ are located on Curve 2, we 
need a family (uncountably infinite) of such curves covering the whole unit (twodimensional) space to do the job.

Property 7 (MONOTONICITY). (i) $F\left(x_{1}, y_{1}, x_{2}\right)$ monotonically increases in $x_{2}$; (ii) $F\left(x_{1}, y_{1}, x_{2}\right)$ monotonically increases in $y_{1}$ and decreases in $x_{1}$ except when it has already reached its limiting points (e.g., when $y_{2}=x_{2}=1$ ).

The compelling nature of this requirement is obvious. This property makes each and every function $y=f^{i}(x)$ strictly increasing in $x$. Geometrically, each curve is upward sloping. Moreover, they do not intersect except at the extreme points $(0,0)$ and $(1,1)$.

Property 8 (Differentiability). Each and every function $y=f^{i}(x)$ is continuous and differentiable at all points.

If $x$ changes marginally (i.e., infinitesimally), we do not expect $y$ to jump up or down to be an equivalent change. Also, we do not expect the rate of change in $y$ to jump.

Property 9 (Complementarity). $F\left(1-x_{1}, 1-y_{1}, 1-x_{2}\right)=1-F\left(x_{1}, y_{1}, x_{2}\right)$.

If $x$ is the probability/percentage of event $X$, then $1-x$ is the probability/percentage of non- $X$. Similarly for $y$. For example, suppose male employment rate changes from $90 \%$ to $99 \%$, and we believe that female employment rate, starting from $70 \%$, has to increase to, say, $77 \%$ to be an equivalent change. Then we must also accept that, when maie unemployment rate changes from $10 \%$ to $1 \%$, the equivalent change for female unemployment, starting from $30 \%$, is to go to $23 \%$. This example also illustrates the unreasonableness of taking proportionate change, even if this does not exceed the limits of $0 \%$ and $100 \%$. While it may look not too unreasonable to take a $1 / 10$ increase in female employment from $70 \%$ to $77 \%$ as equivalent to a $1 / 10$ increase in male employment from $90 \%$ to $99 \%$, it is clearly unreasonable to regard a fall from $10 \%$ to $1 \%$ as equivalent to a fall from $30 \%$ to $23 \%$.

In terms of $y=f^{i}(x)$, Property 9 requires each and every $f^{i}(x)$ that passes below/above the $45^{\circ}$ line $y=x$ (e.g., Curve $2 / 2^{\prime}$ in Fig. 1) to have its complementary curve (e.g., Curve $2^{\prime} / 2$ in Fig. 1) that passes above/below the $45^{\circ}$ line as an inverted mirror image of each other as shown in Fig. 1.

While Property 3 above requires the interchangeability of subscripts (which may stand for time periods, regions, etc.) 1 and 2, Property 10 below requires the interchangeability of $x$ and $y$. A pair of variables should not receive different treatment whether we call the first one $x$ or $y$.

Property 10 (ANonymity). $F\left\{y_{1}, x_{1}, F\left(x_{1}, y_{1}, x_{2}\right)\right\}=x_{2}$.

It is easy to see that this property requires each $f^{i}$ (e.g., Curve 3 in Fig. 2) to have its corresponding one that is a mirror image of itself along the $45^{\circ}$ line as shown in Fig. 2.

Together, Complementarity and Anonymity implies Symmetry, that each and every $y=f^{i}(x)$ must define a curve in the unit space that is symmetrical along the negative $45^{\circ}$ line, as shown in Fig. 3, where the segment $0 A$ is the mirror image of the segment $B A$ (with the mirror along the dotted negative $45^{\circ}$ line). This must be 


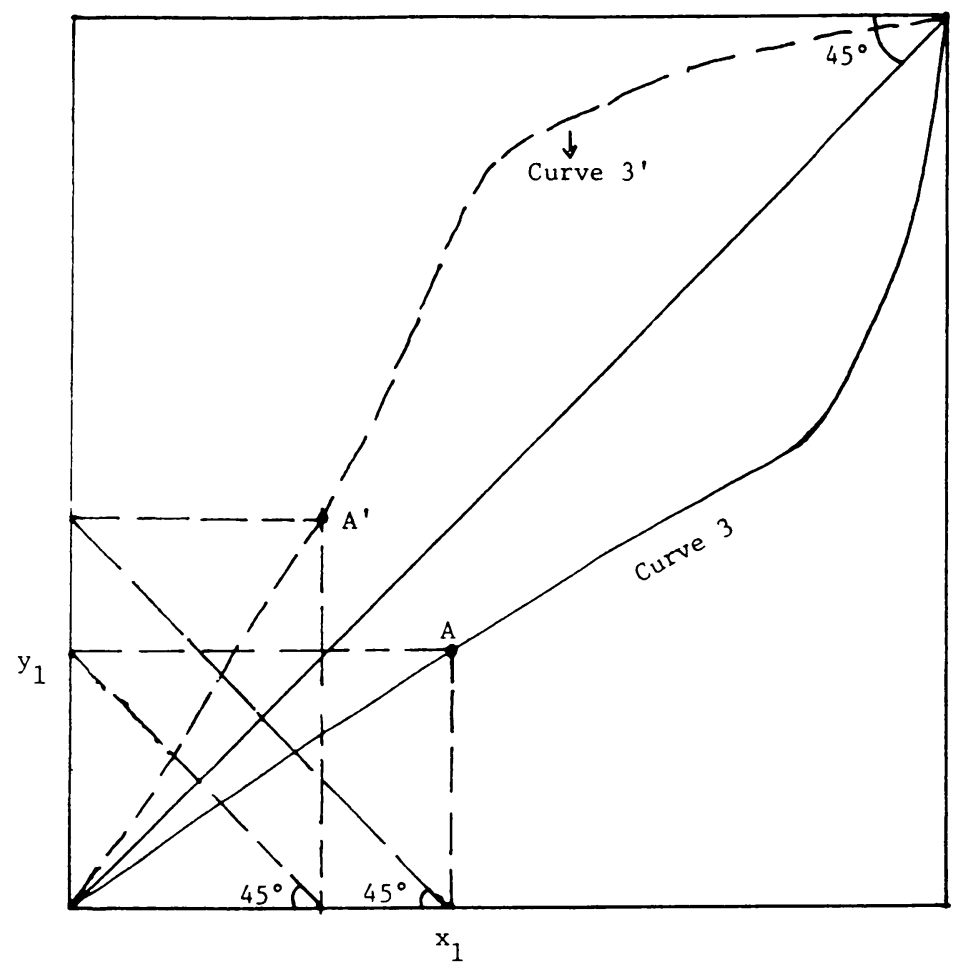

FIG. 2

so because we cannot have the complementary curve being the inverted mirror image (as required by Property 9) and the corresponding curve being the (uninverted) mirror image (as required by Property 10) at the same time unless each curve defined by $y=f^{i}(x)$ is itself symmetrical around the negtive $45^{\circ}$ line. (From Properties 2 and 7 , we cannot have the complementary curve and the corresponding curve intersecting each other.) In fact, even if Symmetry did not follow from other reasonable properties, it would be a reasonable requirement to impose itself.

Property 11 (Transitivity). $F\left(z_{1}, y_{1}, z_{2}\right)=y_{2}$ for all $y_{2}=F\left(x_{1}, y_{1}, x_{2}\right)$ and $z_{2}=F\left(x_{1}, z_{1}, x_{2}\right)$.

If the change in $y$ from $y_{1}$ to $y_{2}$ is equivalent to the change in $x$ from $x_{1}$ to $x_{2}$ and the change in $z$ from $z_{1}$ to $z_{2}$ is also equivalent to the same change in $x$ from $x_{1}$ to $x_{2}$, then obviously the change in $y$ (from $y_{1}$ to $y_{2}$ ) should also be equivalent to the change in $z$ (from $z_{1}$ to $z_{2}$ ). Graphically, Transitivity is illustrated in Fig. 4. If point $A\left(x_{1}, y_{1}\right)$ and point $B\left(x_{2}, y_{2}\right)$ lie on the same curve, and point $C\left(x_{1}, z_{1}\right)$ and point $D\left(x_{2}, z_{2}\right)$ also lie on the same curve (possibly different from the previous curve), then point $E\left(z_{1}, y_{1}\right)$ and point $F\left(z_{2}, y_{2}\right)$ must also lie on the same curve (possibly different from the previous two curves). Figure 5 illustrates the violation of Transitivity. Here, points $E$ and $F$ cannot lie on the same curve since these curves cannot intersect each other (from Monotonicity). 


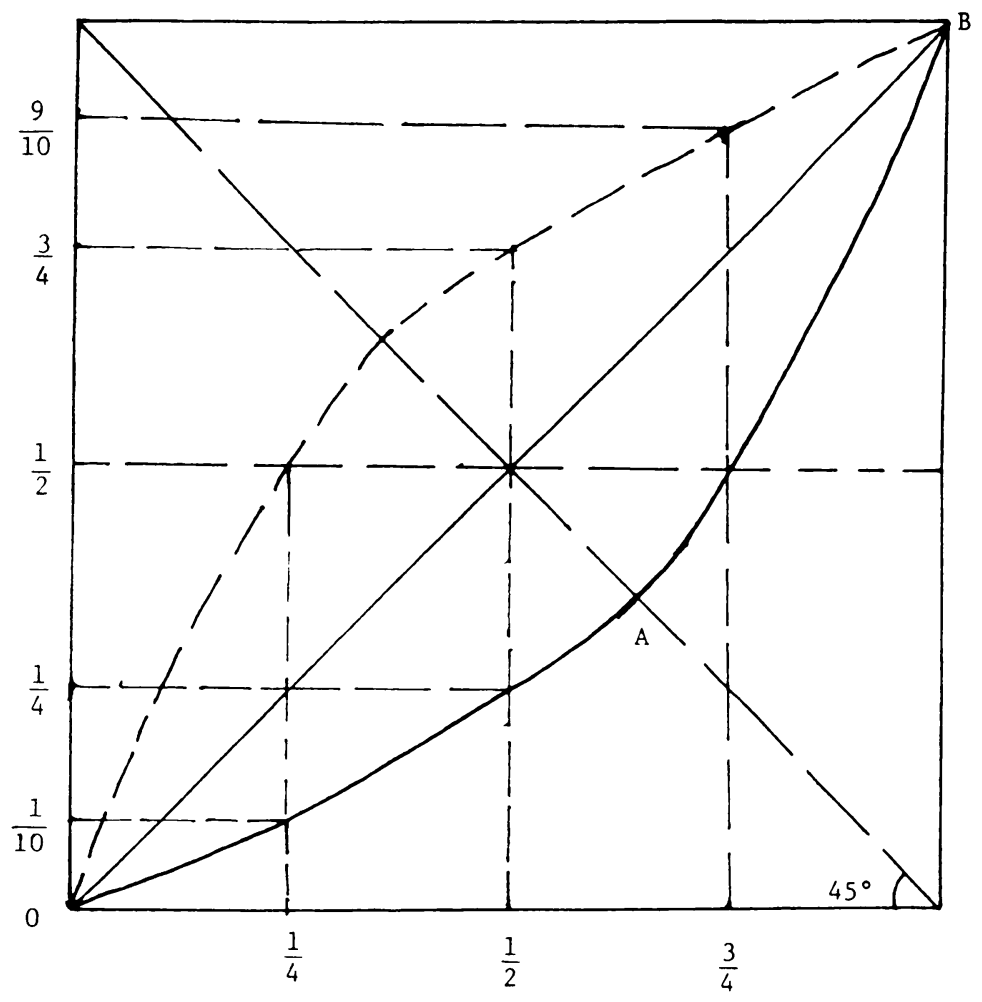

FIG. 3

III. A function meeting all requirements. From the discussion above, the problem of finding a function $y_{2}=F\left(x_{1}, y_{1}, x_{2}\right)$ satisfying our requirements can be transformed into one of finding a family of functions $y=f^{i}(x)$ for $0 \leq x, y \leq 1$ that is strictly increasing in $x$, symmetrical around the negative $45^{\circ}$ line, all passing through $(0,0)$ and $(1,1)$, nonintersecting between any two, and covering the whole two-dimensional unit space.

A function $y_{2}=F\left(x_{1}, y_{1}, x_{2}\right)$ satisfying all our requirements is given herewith:

$$
y_{2}=\frac{x_{2} y_{1}\left(1-x_{1}\right)}{x_{1}\left(1-x_{2}\right)+y_{1}\left(x_{2}-x_{1}\right)} \text {. }
$$

In terms of a family of functions (curves), we have

$$
y=\frac{1 \pm \sqrt{1+4 \alpha}}{2}-\frac{\alpha}{x-(1 \mp \sqrt{1+4 \alpha}) / 2},
$$

where $\alpha$ is a positive parameter varying between zero and infinity to generate different curves covering the unit space. We have $y=x$ as the limit when $\alpha$ approaches infirity. Let us call this family of curves the equivalent hyperbolae (for positive proper fractions, i.e., percentages or probabilities between zero and one); so named because it is in fact derived from the rectangular hyperbolae $x y=-\alpha$ by vertical and horizontal shifts to ensure that each hyperbola always passes through the points $(0,0)$ and $(1,1)$. This can be seen by noting that if we substitute $x=0 \quad(x=1)$ into $\left(1^{\prime}\right)$, we have $y=0 \quad(y=1)$ for all $\alpha$. 


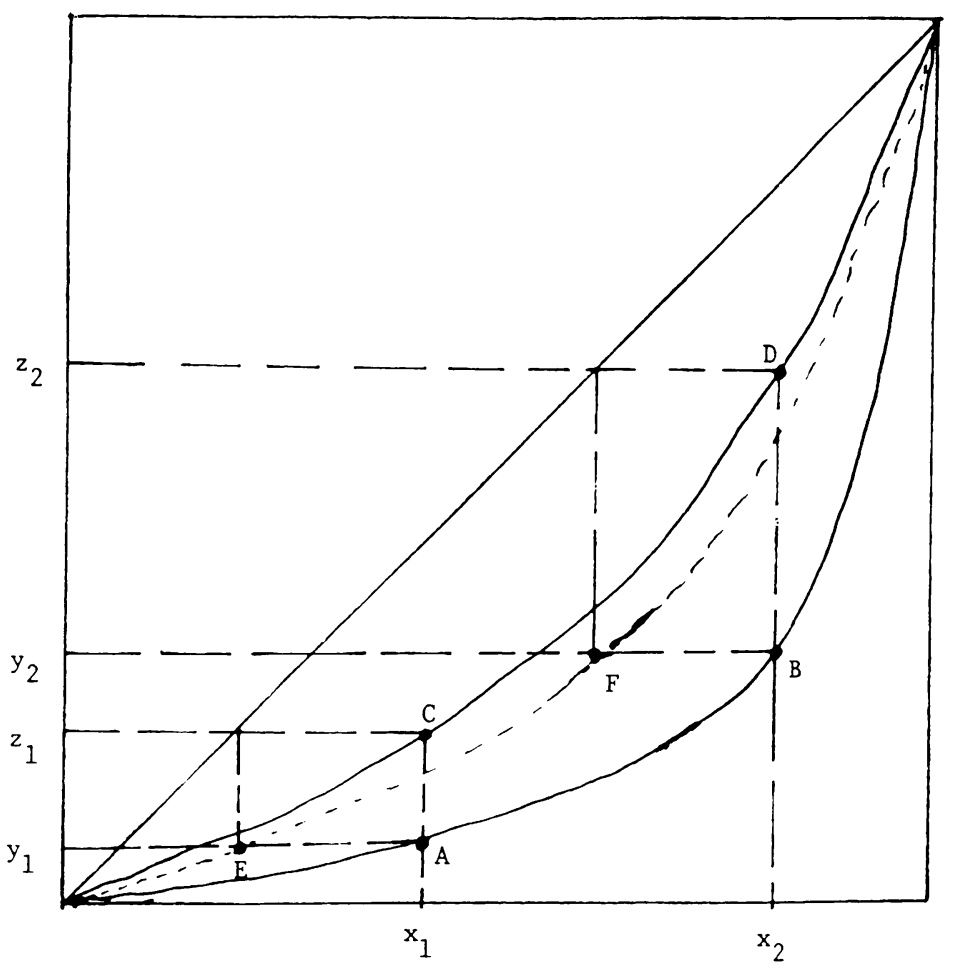

FIG. 4

The rectangular hyperbola $x y=-\alpha$ is chosen to start with because it is upward sloping, continuous, smooth, and infinitely differentiable, and more importantly, symmetrical around the negative $45^{\circ}$ line. Since the vertical and horizontal shifts are designed to make each curve passing through the extreme points $(0,0)$ and $(1,1)$, each curve remains symmetrical around the negative $45^{\circ}$ line even after the shifts (otherwise it cannot pass through both these extreme points). We may also check for Symmetry of Eq. $\left(1^{\prime}\right)$ by confirming that Eq. $\left(1^{\prime}\right)$ satisfies $f\{1-f(x)\}=1-x$. It can also be confirmed that $d y / d x=1$ at the value of $x$ satisfying $y=1-x$ (i.e., the negative $45^{\circ}$ line).

In Eq. $\left(1^{\prime}\right)$, the \pm and $\mp$ sign means that, for each value of $\alpha$, two curves are defined, the first by taking the upper (i.e., first + and then - ) sequence and the second by taking the lower (i.e., first - and then + ) sequence. The first curve lies above the $45^{\circ}$ line and the second, its corresponding and complementary curve, lies below the $45^{\circ}$ line. For example, with $\alpha=3 / 4$, the two curves are those shown in Fig. 3. This example means that, e.g., if we start with $x_{1}=25 \%, y_{1}=10 \%$, and $x$ increases to $50 \%, y$ has to increase to $25 \%$ to register an equivalent change.

For any specific values of $x_{1}$ and $y_{1}$ (between zero and one), we substitute these values into Eq. $\left(1^{\prime}\right)$ to solve for the specific value of $\alpha$. (If $y_{1}$ is larger/smaller than $x_{1}$, take the upper/lower sequence. With $y_{1}=x_{1}$, take $\alpha=\infty$ and Eq. $\left(1^{\prime}\right)$ becomes $y=x$.) With this specific value of $\alpha$, we can then use Eq. $\left(1^{\prime}\right)$ again 


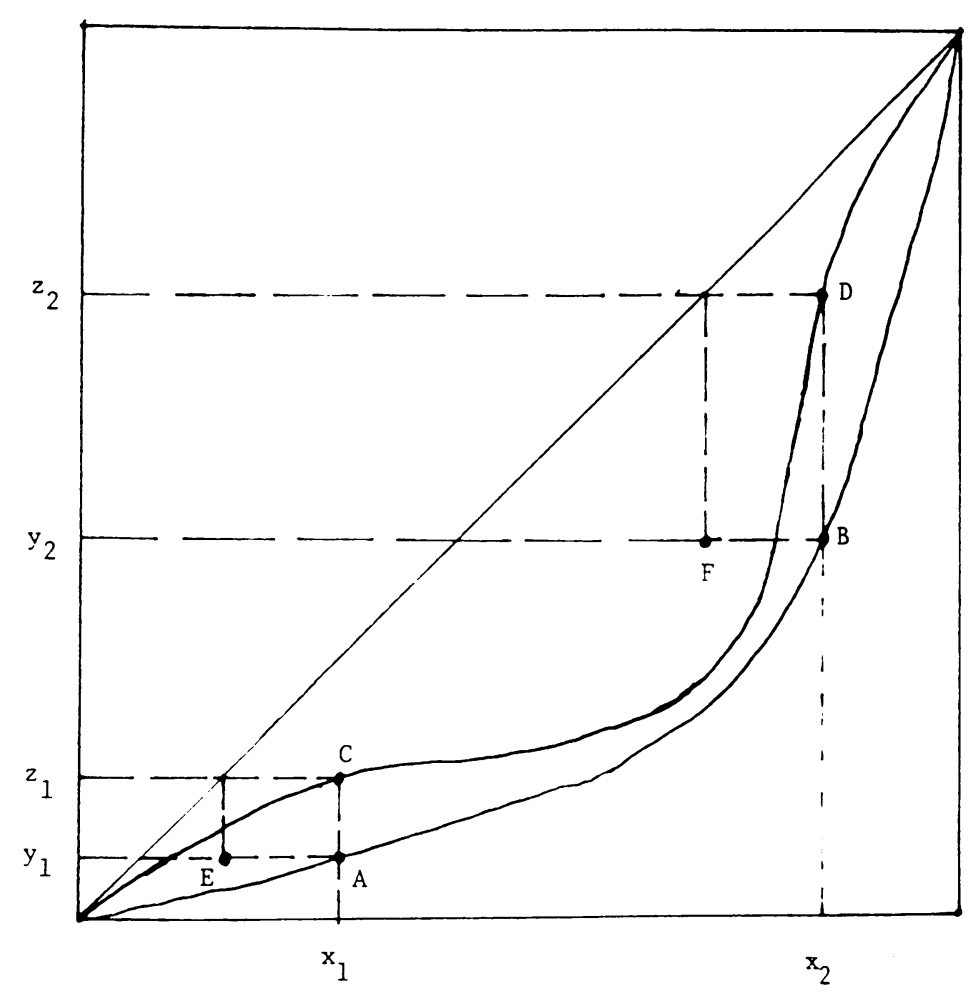

FIG. 5

to solve for $y_{2}$ for any value of $x_{2}$. Using this process, we obtain one and only one value of $y_{2}$ for each combination of $x_{1}, y_{1}$, and $x_{2}$. To see that this gives us Eq. (1), note that it can be written as

$$
\begin{aligned}
y_{2} & =\frac{1+E}{2}-\frac{\left(E^{2}-1\right) / 4}{x_{2}-(1-E) / 2}=\frac{(1+E) x}{2 x+E-1} \\
& =\frac{x_{2} y_{1}\left(1-x_{1}\right)}{x_{1}\left(1-x_{2}\right)+y_{1}\left(x_{2}-x_{1}\right)},
\end{aligned}
$$

where $E \equiv\left(2 x_{1} y_{1}-x_{1}-y_{1}\right) /\left(x_{1}-y_{1}\right)=\sqrt{1+4 \alpha}$, and $\alpha=\left(E^{2}-1\right) / 4$.

It is not difficult to check that the equivalence function (for proper fractions) defined by Eq. (1) or Eq. ( $\left.1^{\prime}\right)$ satisfies all Properties $1-11$ in the preceding section. Since Transitivity seems to be the most demanding property, its satisfaction by the above proposed function is shown in Appendix A. Readers can easily check for the satisfaction of other properties. For example, the satisfaction of Monotonicity can be confirmed by evaluating

$$
\begin{aligned}
& \partial y_{2} / \partial x_{2}=x_{1} y_{1}\left(1-x_{1}\right)\left(1-y_{1}\right) / H^{2}>0 \\
& \partial y_{2} / \partial y_{1}=x_{1} x_{2}\left(1-x_{1}\right)\left(1-x_{2}\right) / H^{2}>0 \\
& \partial y_{2} / \partial x_{1}=-x_{2} y_{1}\left(1-y_{1}\right)\left(1-x_{2}\right) / H^{2}<0
\end{aligned}
$$


where $H \equiv x_{1}\left(1-x_{2}\right)+y_{1}\left(x_{2}-x_{1}\right)$. Symmetry can be seen by rewriting Eq. (1) as

$$
x_{1} x_{2}\left(y_{1}-y_{2}\right)+x_{1} y_{2}=y_{1} y_{2}\left(x_{1}-x_{2}\right)+x_{2} y_{1} \text {. }
$$

Complementarity is shown by substituting $1-x_{1}, 1-y_{1}, 1-x_{2}$, and $1-y_{2}$ respectively in place of $x_{1}, y_{1}, x_{2}, y_{2}$ into Eq. (1) which is then shown to simplify back into Eq. (1).

IV. Uniqueness and further research. Though the function defined above satisfies all our required properties and also appears to be a reasonable function for defining an equivalent change for (positive) proper fractions, there is still the problem whether it is the only function satisfying these requirements. I do not have a conclusive answer to this question. However, intuitively, it looks possible for other (possibly infinite number of) functions to exist which satisfy all Properties 1-10. This is illustrated in Fig. 6 where the solid Curve 1 is as defined by Eq. $\left(1^{\prime}\right)$. It does not seem impossible to construct a curve that lies slightly above Curve 1 in some segments and below it in other in such a way as to be itself symmetrical around the negative $45^{\circ}$ line as shown by the broken curve in Fig. 6. As we go to another curve defined by Eq. (1), we make the difference between the solid and the broken curve smaller and smaller and approaching zero as the solid curve approaches either the $45^{\circ}$ line or the axes. It appears that the family of broken curves thus constructed also defines a function $y_{2}=G\left(x_{1}, y_{1}, x_{2}\right)$ satisfying Properties 1-10. Moreover, by making the difference between the broken and solid curves differing by different amounts and differing over different segments, it appears that there exists an infinity of such functions.

It is less clear whether a family of curves defined in the preceding paragraph can also satisfy Property 11 (Transitivity). If the answer is negative, an obvious theme for further study is to prove the uniqueness (under Properties 1-11) of the Equivalence Function (Eq. (1)). If the answer is positive, several issues for further research also suggest themselves. First, the discovery of the precise solutions of functions satisfying Properties 1-11. Second, the comparison of these functions with the one we obtained above to see which one has the best claim to provide the general equivalent changes for proper fractions. In this connection, while I have no proofs for the following, I strongly suspect that: (1) our solution contained in Eq. (1) provides a natural and best solution for equivalent changes in the general sense (Sec. I); (2) all other solutions are variants based on this basic solution.

It should be noted, however, that we cannot derive a variant by, e.g., adding to a curve defined by Eq. $\left(1^{\prime}\right)$ a value $(\Delta y)$ to $y$, as defined by the curve $\Delta y$ in Fig. 7 that is symmetrical around the point $1 / 2$. This method does not work because the resulting $y+\Delta y$ is no longer symmetrical around the negative $45^{\circ}$ line. This can be seen by referring back to Fig. 6 . For the broken curve to be symmetrical, if it cuts the symmetrical solid curve at $\bar{x}$, then it must cut it again at $1-f(\bar{x})$, not at $1-\bar{x}$. But the later intersection point is what would be the case with the symmetrical $\Delta y$ of Fig. 7. More generally, if we subtract (or add) a value equal to $A A^{\prime}$ (Fig. 6) from a point $A$ (when $x=x^{*}$ ) on the solid curve to obtain a point on the broken curve, we do not subtract (or add) the same value from the solid curve at $1-x^{*}$, nor even at $1-f\left(x^{*}\right)$. Rather, it is the horizontal distance $B B^{\prime}$ that should equal 


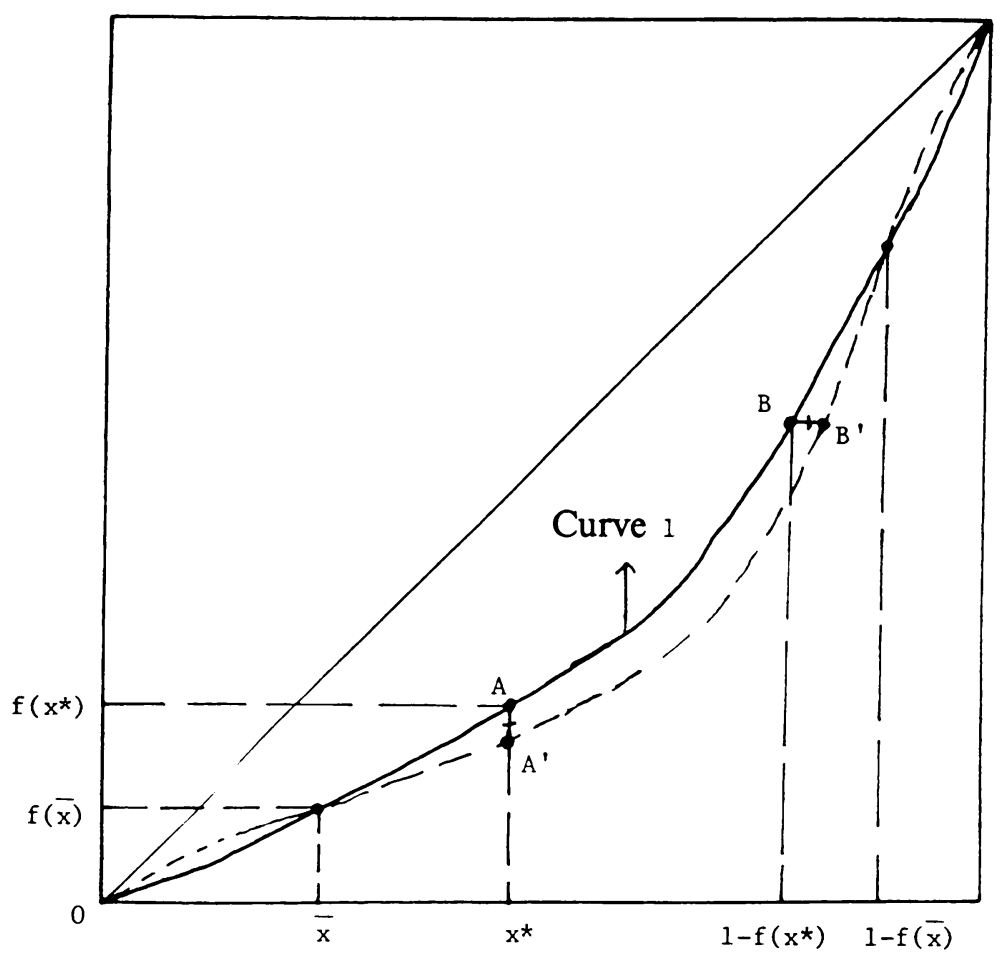

FIG. 6

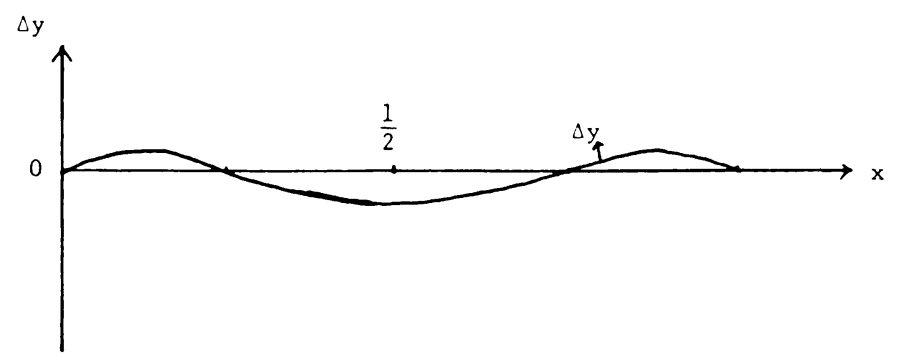

FIG. 7

$A A^{\prime}$, where $B$ is the symmetrical point to $A$, i.e., when $x=1-f\left(x^{*}\right)$.

Third, we may wish to adopt further reasonable properties so as to narrow down the permissible functions defining equivalent changes, preferably to a unique function $y_{2}=F\left(x_{1}, y_{1}, x_{2}\right)$ or a unique family of functions $y=f^{i}(x)$. One such further property is illustrated in Fig. 8. The solid curve is defined by Eq. $\left(1^{\prime}\right)$. For any curve defined by Eq. $\left(1^{\prime}\right)$, lying above/below the $45^{\circ}$ line, $d y / d x$ is decreasing/increasing throughout. This is so because we have, from Eq. $\left(1^{\prime}\right)$,

$$
\frac{d^{2} y}{d x^{2}}=\frac{-2 \alpha}{\{x-(1 \mp \sqrt{1+4 \alpha}) / 2\}^{3}},
$$




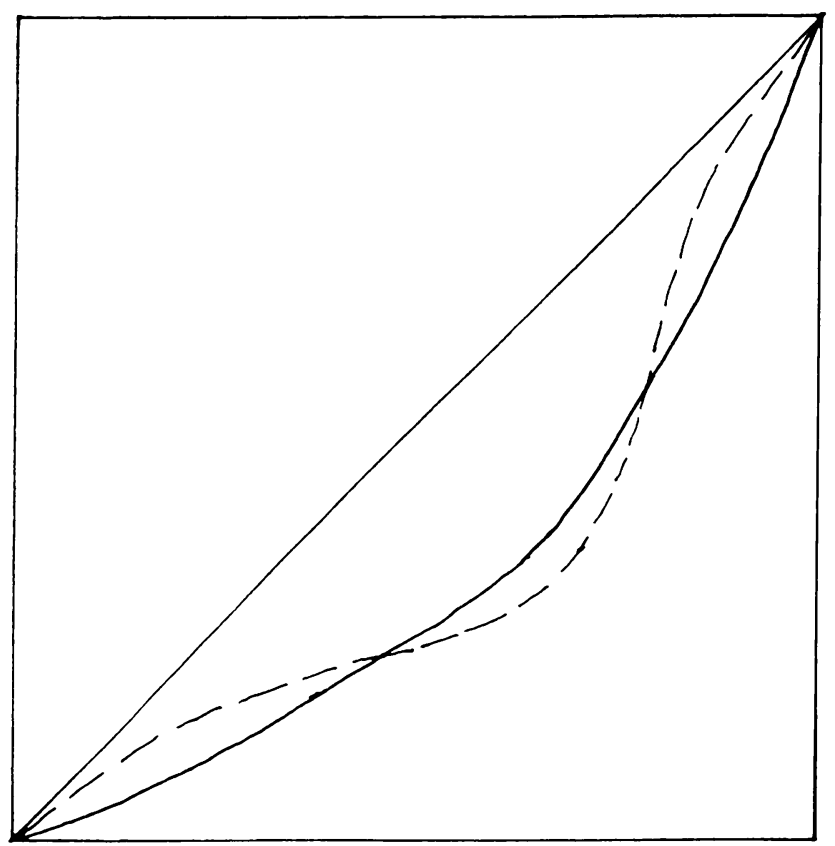

FIG. 8

which is negative/positive for all $x$ (between zero and one) and all $\alpha$ (between zero and infinity) if we take the upper/lower (negative/positive) sign. For higher order derivatives, we have

$$
\frac{d^{n} y}{d x^{n}}=\frac{-(-1)^{n} n ! \alpha}{(x-(1 \mp \sqrt{1+4 \alpha}) / 2)^{n+1}},
$$

which is positive throughout for all odd $n$ and negative/positive throughout for all even $n$ for curves lying above/below the $45^{\circ}$ line. In terms of Eq. (1), we have

$$
d^{n} y_{2} / d x_{2}^{n}=x_{1} y_{1}\left(1-x_{1}\right)\left(1-y_{1}\right)\left(x_{1}-y_{1}\right)^{n-1} / H^{n+1},
$$

which gives us the same result.

That $d y / d x$ should be decreasing/increasing for curves lying above/below the $45^{\circ}$ line reflects the following reasonable requirement. If $y_{1}<x_{1}$ and both are small, an increase in $x$ only necessitates a small increase in $y$ to register an equivalent increase. But as both variables increase equivalently together, the more $x$ approaches 1 , the less scope there is for further increase in $x$. This is less so for $y$ since it starts at a lower value to begin with. Thus, as $x$ increases, it calls for higher and higher values of $d y / d x$ for equivalent increases. We may thus formally require

Property 12 (MONOTONICITY IN $d y / d x$ ). If $x_{1}$ is larger/smaller than $y_{1}$, then $d y_{2} / d x_{2}$ monotonically increases/decreases in $x_{2}$.

The broken curve in Fig. 8 satisfies Properties $1-10$ but does not satisfy Property 12. However, I am not sure whether Property 11 is satisfied and whether Properties 1-12 are sufficient to ensure uniqueness. If not, we may have to add further 
requirements on the monotonicity of $d^{2} y / d x^{2}$ and possibly higher order derivatives and/or other reasonable requirements.

Being myself an economist rather than a mathematician or logician, I leave the solutions of the above problems to more qualified researchers. Whatever the outcome of these further studies, I am confident that Eq. (1) provides one (if not the only) acceptable function for equivalent changes that may lead to widespread practical application in many fields. For example, a teacher adjusting a student's mark from $45 \%$ to $50 \%$ would like to know how to adjust the marks of other students equivalently. An accepted equivalence function could be built into a functional key in a calculator for handy use.

Appendix A: The equivalence function satisfies transitivity. In this appendix, it is shown that the equivalence function (1) satisfies Transitivity.

First, check for Transitivity by substituting specific numerical values (between zero and one) into (1). Take $x_{1}=1 / 4, y_{1}=1 / 7, x_{2}=1 / 2$. Equation (1) gives $y_{2}=1 / 3$. This means that, if $x$ increases from $1 / 4$ to $1 / 2$, starting from $y_{1}=1 / 7, y$ has to increase to $y_{2}=1 / 3$ to register an equivalent change according to our proposed equivalence function.

Now take $z_{1}=1 / 10$. Substitute this (in place of $y_{1}$ ) and $x_{1}=1 / 4, x_{2}=1 / 2$ into (1), yielding $z_{2}$ (in place of $y_{2}$ ) $=1 / 4$. This means that if $z$ increases from $1 / 10$ to $1 / 4$, this is also equivalent to an increase in $x$ from $1 / 4$ to $1 / 2$.

If the equivalence function based on Eq. (1) satisfies Transitivity, we must have the change in $y$ (from $1 / 7$ to $1 / 3$ ) also equivalent to the change in $z$ (from $1 / 10$ to $1 / 4$ ) according to the same equivalence function. To check for this, we now take the variable $x$ in Eq. (1) to stand for $z$. Substitute $z_{1}$ (as $\left.x_{1}\right)=1 / 10, y_{1}=1 / 7$, and $z_{2}\left(\right.$ as $\left.x_{2}\right)=1 / 4$ in Eq. (1), obtaining $y_{2}=1 / 3$, confirming that the change in $y$ is equivalent to the change in $z$ according to the equivalence function.

While the above demonstration is satisfying, it does not constitute a general proof. We might just be lucky that Transitivity holds only at the specific combination of variables we have chosen. Since there are uncountable possibilities for each variable, to say that the number of possible combinations is astronomical is, mathematically speaking, an understatement of the century. Let us use a more general method.

We wish to show that, if we have

$$
\begin{aligned}
& y_{2}=\frac{x_{2} y_{1}\left(1-x_{1}\right)}{x_{1}\left(1-x_{2}\right)+y_{1}\left(x_{2}-x_{1}\right)}, \\
& z_{2}=\frac{x_{2} z_{1}\left(1-x_{1}\right)}{x_{1}\left(1-x_{2}\right)+z_{1}\left(x_{2}-x_{1}\right)},
\end{aligned}
$$

we must have

$$
y_{2}=\frac{z_{2} y_{1}\left(1-z_{1}\right)}{z_{1}\left(1-z_{2}\right)+y_{1}\left(z_{2}-z_{1}\right)} \text {. }
$$

Rearrange (A2) to give

$$
x_{2}=\frac{z_{2} x_{1}\left(1-z_{1}\right)}{z_{1}\left(1-z_{2}\right)+x_{1}\left(z_{2}-z_{1}\right)} .
$$

Substitute $x_{2}$ from (A4) into (A1) to obtain, after simplification, (A3). Q.E.D. 
We have thus shown that our proposed equivalence function based on Eq. (1) satisfies Transitivity.

Acknowledgment. I am grateful to Ken Davis and Victor Kacala of the University of Melbourne for stimulting and fruitful discussion.

\section{REFERENCES}

[1] A. W. F. Edwards, The measure of association in a $2 \times 2$ table, Roy. Statist. Soc. J. A 126, 109-114 (1963)

[2] L. A. Goodman and W. H. Kruskal, Measures of Association for Cross Classifications, SpringerVerlag, New York, 1979 